\title{
RECTILINEAR CONGRUENCES WHOSE DEVELOPABLES INTERSECT A SURFACE IN ITS LINES OF CURVATURE
}

\author{
C. E. SPRINGER
}

Introduction. It is well known that the developable surfaces of the congruence of normals to a surface intersect the surface in its lines of curvature. One may inquire if there exist congruences other than the congruence of normals to a surface the developables of which intersect the surface in its lines of curvature. It is the chief aim of this paper to give an affirmative answer to this query. The exhibition of a congruence of the required variety depends upon the solution of a partial differential equation of Laplace-a circumstance which occurs frequently in problems of differential geometry.

The notation employed here is that of Eisenhart, ${ }^{1}$ with the exception that $\Gamma_{\beta \gamma}^{\alpha}$ will be used for the Christoffel symbol of the second kind. Greek letters will take the range 1, 2, and Latin letters the range $1,2,3$. The convention of the tensor analysis as to summation on repeated indices will be observed.

1. Analytical development. Consider a surface $S$ represented by $x^{i}=x^{i}\left(u^{1}, u^{2}\right)(i=1,2,3)$ referred to a rectangular cartesian system of coordinates. The functions $x^{i}\left(u^{1}, u^{2}\right)$, together with their partial derivatives to the second order, are understood to be continuous at any point $P$ of the surface. A unique line $\lambda$ of a congruence $\Lambda$ is determined at each point $P$ of the surface $S$ by the direction cosines

$$
\lambda^{i}=\lambda^{i}\left(u^{1}, u^{2}\right), \quad \quad \lambda^{i} \lambda^{i}=1,
$$

where the functions $\lambda^{i}$ and their first partial derivatives are continuous at points of the surface under consideration.

The functions $\lambda^{i}$ may be expressed in terms of the direction numbers $x^{i}, \alpha(\alpha=1,2)$ of the tangents to the coordinate curves on the surface through $P$, and the direction cosines $X^{i}$ of the normal to the surface at $P$. Thus,

$$
\lambda^{i}=p^{\alpha} x^{i}, \alpha+q X^{i},
$$

where $p^{\alpha}$ are the contravariant components of a vector in the surface at $P, q$ is a positive scalar function, and $x^{i}, \alpha$ denotes covariant 1945.

Presented to the Society, November 24, 1945; received by the editors June 20,

1 Eisenhart, Differential geometry, Princeton University Press, 1940. 
differentiation of $x^{i}$ with respect to $u^{\alpha}$, based on the first fundamental tensor $g_{\alpha \beta} \equiv x^{i},{ }_{\alpha} x^{i},{ }_{\beta}$ of the surface $S$.

On using (2) and the second of equations (1), there results

$$
\lambda^{i} \lambda^{i} \equiv\left(p^{\alpha} x^{i}{ }_{, \alpha}+q X^{i}\right)\left(p^{\beta} x^{i}{ }_{, \beta}+q X^{i}\right) \equiv g_{\alpha \beta} p^{\alpha} p^{\beta}+q^{2}=1 .
$$

If $\theta$ is the angle between the normal to $S$ at $P$ and the line $\lambda$ of the congruence $\Lambda$ at $P$, it follows from (3) that

$$
\cos \theta \equiv \lambda^{i} X^{i}=q \text {. }
$$

Thus, from equation (3), it is seen that the length of the vector with contravariant components $p^{\alpha}$ is $\sin \theta$. That is, the last of equations (3) assumes the form

$$
p_{\alpha} p^{\alpha}=\sin ^{2} \theta
$$

It may be noticed here that the angle $\phi$ between the line $\lambda$ of the congruence $\Lambda$ at $P$ and the direction $d x^{i} / d s$ of any curve $C: u^{\alpha}=u^{\alpha}(s)$ through $P$ on $S$ is given by

$$
\cos \phi \equiv \lambda^{i} d x^{i} / d s=\left(p^{\alpha} x^{i}, \alpha+q X^{i}\right) x^{i}{ }_{\beta} d u^{\beta} / d s=g_{\alpha \beta} p^{\alpha} d u^{\beta} / d s,
$$

where $s$ denotes arc length along $C$.

Covariant differentiation of $\lambda^{i}$ in equation (2) gives

$$
\lambda^{i}{ }_{, \beta}=p^{\alpha} x^{i}{ }_{, \alpha \beta}+q X^{i}{ }_{, \beta}+x^{i}{ }_{, \alpha} p^{\alpha}{ }_{, \beta}+X^{i} q_{, \beta},
$$

which, by means of the Gauss and Weingarten equations ${ }^{2}$

$$
x^{i}{ }_{, \alpha \beta}=d_{\alpha \beta} X^{i}, \quad X^{i}{ }_{, \beta}=-d_{\beta \gamma} g^{\gamma \delta} x^{i}, \delta,
$$

can be written in the form

$$
\lambda^{i}, \alpha=\mu_{\alpha}^{\gamma} x^{i}, \gamma+\nu_{\alpha} X^{i},
$$

where, in turn, $\mu^{\gamma}{ }_{\alpha}$ and $\nu_{\alpha}$ are defined by

$$
\mu^{\gamma}{ }_{\alpha} \equiv p^{\gamma}{ }_{, \alpha}-q d_{\alpha \sigma} g^{\sigma \gamma}, \quad \nu_{\alpha} \equiv q_{, \alpha}+p^{\beta} d_{\alpha \beta} .
$$

Differentiation of $\lambda^{i} \lambda^{i}=1$ yields $\lambda^{i} \lambda^{i}{ }_{, \alpha}=0$, which can be written, by use of (2) and (4), in the form

or

$$
\left(p^{\sigma} x^{i}, \sigma+q X^{i}\right)\left(\mu^{\gamma}{ }_{\alpha} x^{i}{ }_{, \gamma}+\nu_{\alpha} X^{i}\right)=0,
$$

$$
p_{\gamma} \mu_{\alpha}^{\gamma}+q \nu_{\alpha}=0
$$$$
(\alpha=1,2) \text {. }
$$

Because $q$ does not vanish, $h_{\gamma}$ may be written for $p_{\gamma} / q$, so that equation (6) takes the form

$$
\nu_{\alpha}=-h_{\gamma} \mu_{\alpha} \quad(\alpha=1,2) .
$$

${ }^{2}$ Eisenhart, loc. cit. pp. 216, 217. 
2. Developables of the congruence and their intersections with the surface. In order to arrive at the differential equation of the two oneparameter families of curves on the surface $S$ in which the developables of the congruence $\Lambda$ intersect the surface, take a point with coordinates $x^{i}+t \lambda^{i}$ on the line $\lambda$ through $P$ on $S$, and let $P$ move along a curve $C: u^{\alpha}=u^{\alpha}(s)$ on $S$ for which the point with coordinates $x^{i}+t \lambda^{i}$ will describe a curve tangent to the line $\lambda$. This requires that

$$
d\left(x^{i}+t \lambda^{i}\right) \equiv d x^{i}+t d \lambda^{i}+\lambda^{i} d t=m \lambda^{i},
$$

where $m$ is to be determined. Multiplication of equation (8) by $\lambda^{i}$ and use of $\lambda^{i} \lambda^{i}=1$ show that $m=d t+\lambda^{i} d x^{i}$, by means of which equation (8) becomes

$$
d x^{i}+t d \lambda^{i}=\lambda^{i}\left(\lambda^{k} d x^{k}\right)
$$

or

$$
\left[x^{i}, \alpha+t \lambda^{i, \alpha}-\lambda^{i}\left(\lambda^{k} x^{k}{ }_{, \alpha}\right)\right] d u^{\alpha}=0 .
$$

From equations (2), (4), (7), it is observed that

$$
\begin{gathered}
\lambda^{i} x_{, \alpha}^{i}=p^{\sigma} g_{\sigma \alpha}=p_{\alpha}, \\
\lambda^{i}{ }_{, \alpha} \lambda^{i}{ }_{, \beta}=\mu_{\alpha}^{\sigma} \mu^{\gamma}{ }_{\beta} g_{\sigma \tau}+\nu_{\alpha} \nu_{\beta}=\mu_{\alpha}^{\sigma} \mu_{\beta}{ }_{\beta}\left(g_{\sigma \tau}+h_{\sigma} h_{\tau}\right), \\
\lambda^{i}{ }_{, \alpha} x^{i}{ }_{, \beta}=\mu^{\gamma}{ }_{\alpha} x^{i}{ }_{, \gamma} x^{i}{ }_{, \beta}=\mu^{\gamma}{ }_{\alpha} g_{\gamma \beta}=\mu_{\beta \alpha} .
\end{gathered}
$$

Multiplication of equation (9) by $\lambda^{i}, \beta$, together with use of equations (10), (11), (12), yields

$$
\left(\mu_{\alpha \beta}+t G_{\alpha \beta}\right) d u^{\alpha}=0
$$

where $G_{\alpha \beta}$ is an abbreviation for

$$
\lambda^{i}{ }_{, \alpha} \lambda^{i}, \beta \equiv \mu_{\alpha}^{\sigma} \mu_{\beta}^{\tau}\left(g_{\sigma \tau}+h_{\sigma} h_{\tau}\right) .
$$

Elimination of the parameter $t$ from equations (13) gives the differential equation

$$
e^{\gamma \delta} \mu_{\alpha \gamma} G_{\beta \delta} d u^{\alpha} d u^{\beta}=0
$$

in which $e^{\gamma \delta}$ is defined as follows : $e^{12}=1, e^{21}=-1, e^{11}=e^{22}=0$. The curves on the surface which satisfy equation (15) constitute the net of curves in which the developables of the congruence intersect the surface. This net will be referred to as the intersector net.

3. Particular congruences. It will be shown next that equation (15) can be written in the form

$$
e_{\alpha \gamma}\left(\mu_{\beta}^{\gamma}-h^{\gamma} \nu_{\beta}\right) d u^{\alpha} d u^{\beta}=0 .
$$


From the definition of $G_{\alpha \beta}$ in (14) it follows that

$$
\begin{aligned}
e^{\gamma \delta} \mu_{\alpha \gamma} G_{\beta \delta} & \equiv e^{\gamma \delta} \mu_{\alpha \gamma} \mu_{\beta}^{\sigma} \mu^{\tau}{ }_{\delta}\left(g_{\alpha \tau}+h_{\sigma} h_{\tau}\right) \\
& \equiv e^{\gamma \delta} \mu^{\epsilon}{ }_{\gamma} g_{\alpha \epsilon} \mu^{\sigma}{ }_{\beta} \mu^{\tau} \delta\left(g_{\sigma \tau}+h_{\sigma} h^{\rho} g_{\tau \rho}\right) .
\end{aligned}
$$

By use of the relations $e^{\gamma \delta} \mu^{\epsilon}{ }_{\gamma} \mu_{\delta}^{\tau} \equiv \mu e^{\epsilon \tau}, e^{\epsilon \tau} g_{\epsilon \alpha} g_{\sigma \tau} \equiv g e_{\alpha \sigma}$, where $\mu \equiv\left|\mu_{\beta}^{\alpha}\right|$, $g \equiv\left|g_{\alpha \beta}\right|$, and $e_{\alpha \sigma}$ is defined by $e_{12}=1, e_{21}=-1, e_{11}=e_{22}=0$, the last member of (17) takes the form

$$
\mu g e_{\alpha \sigma}\left(\mu_{\beta}^{\sigma}-h^{\sigma} \nu_{\beta}\right) \text {. }
$$

Thus, on rejecting the nonvanishing factors $\mu, g$, equation (16) follows.

A necessary and sufficient condition ${ }^{3}$ that the intersector net given by (16) be a conjugate net is that

$$
e_{\alpha \sigma}\left(\mu_{\beta}^{\sigma}-h^{\sigma} \nu_{\beta}\right) d^{\alpha \beta}=0,
$$

which, by use of (5) and (7), can be written in the form

$$
e_{\sigma \beta}\left(p^{\sigma} / q\right), \alpha d^{\alpha \beta}=0 \text {. }
$$

Thus, a necessary and sufficient condition that the congruence have the property that its intersector net on $S$ be a conjugate net is that the parameters $h^{\sigma}$ of the congruence satisfy the partial differential equation of first order

$$
e_{\sigma \beta} h^{\sigma}{ }_{, \alpha} d^{\alpha \beta}=0 .
$$

The intersector net on $S$ is orthogonal, if, and only if,

$$
e_{\alpha \sigma}\left(\mu_{\beta}^{\sigma}-h^{\sigma} \nu_{\beta}\right) g^{\alpha \beta}=0 .
$$

Again, by use of (5) and (7), and the identical vanishing of the term $e_{\gamma \beta} d_{\alpha \sigma} g^{\sigma \gamma} g^{\alpha \beta}$, it is found that a necessary and sufficient condition for the orthogonality of the intersector net on $S$ is that the parameters $h^{\sigma}$ of the congruence $\Lambda$ satisfy

$$
e_{\gamma \beta}\left(h^{\gamma}, \alpha-d_{\alpha \sigma} h^{\gamma} h^{\sigma}\right) g^{\alpha \beta}=10 .
$$

It is to be observed that if $\Lambda$ is the congruence of normals to $S$ $\left(h^{\sigma} \equiv 0\right)$, then equations (19), (20) are satisfied identically, and equation (16) reduces to the lines of curvature net represented by

$$
e_{\beta \sigma} d_{\alpha \gamma} g^{\gamma \sigma} d u^{\alpha} d u^{\beta}=0 .
$$

The congruence $\Lambda$ is a normal congruence if, and only if, $\mu_{\alpha \beta}=\mu_{\beta \alpha}$. This can be shown as follows. If every line of $\Lambda$ is normal to a surface $\bar{S}$ given by $y^{i}=y^{i}\left(u^{1}, u^{2}\right)$, then $y^{i}=x^{i}+t \lambda^{i}$ and $\lambda^{i} d\left(x^{i}+t \lambda^{i}\right)=0$ identi-

${ }^{3}$ Eisenhart, loc. cit. p. 231. 
cally in $u^{1}, u^{2}$. Because $\lambda^{i} \lambda^{i}{ }_{, \alpha} d u^{\alpha}=0$ and $\lambda^{i}\left(x^{i}, \alpha+\lambda^{i} t_{, \alpha}\right) d u^{\alpha}=0$ for arbitrary direction $d u^{\alpha}$ on $\bar{S}$, it follows that

$$
\lambda^{i} x^{i}{ }_{, 1}+t_{, 1}=0, \quad \lambda^{i} x^{i}{ }_{, 2}+t_{, 2}=0 .
$$

On differentiating the last two equations with respect to $u^{2}, u^{1}$ respectively, and eliminating $\partial^{2} t / \partial u^{1} \partial u^{2}$, there results

$$
x^{i}{ }_{, \alpha} \lambda^{i}{ }_{, \beta}=x^{i}{ }_{, \beta} \lambda^{i}, \alpha,
$$

which, by (4), can be written as

$$
g_{\alpha \gamma} \mu^{\gamma}=g_{\beta \gamma} \mu_{\alpha},
$$

or

$$
\mu_{\alpha \beta}=\mu_{\beta \alpha} .
$$

On multiplying equation (5) by $g_{\beta \gamma}$, it follows from equation (21) that a necessary and sufficient condition for the congruence $\Lambda$ to be a normal congruence is that

$$
p_{\alpha, \beta}=p_{\beta, \alpha}
$$

4. Congruences for which the intersector net coincides with the lines of curvature net. It follows from equation (16) that the intersector net is the parametric net on $S$ if, and only if,

$$
\mu^{\gamma}{ }_{\beta}=h^{\gamma_{\nu_{\beta}}} \quad(\gamma \neq \beta),
$$

which, by means of equations (5) and the fact that $h^{\gamma}=p^{\gamma} / q$, can be expressed in the form

$$
h^{\gamma}{ }_{, \alpha}=\left(g^{\gamma \sigma}+h^{\gamma} h^{\sigma}\right) d_{\alpha \sigma} \quad(\gamma \neq \alpha) .
$$

The first order partial differential equations (23) appear as a twodimensional analogue of an ordinary differential equation of Riccati.

It will now be shown that the two first order partial differential equations (23) in $h^{\gamma}$ become two partial differential equations of the second order by the substitution

$$
h^{\gamma}=-d^{\gamma \sigma} \psi_{, \sigma} \quad(\gamma=1,2),
$$

where $\psi \equiv \log \omega\left(u^{1}, u^{2}\right)$. Covariant differentiation of equations (24) yields

$$
h^{\gamma}{ }_{, \alpha}=-d^{\gamma \sigma} \psi_{, \sigma \alpha}-d^{\gamma \sigma}{ }_{, \alpha} \psi_{, \sigma},
$$

by use of which equations (23) become

$$
-d^{\gamma \sigma} \psi_{, \sigma \alpha}-d^{\gamma \sigma}{ }_{, \alpha} \psi_{, \sigma}=\left(g^{\sigma \gamma}+d^{\sigma \tau} d^{\gamma \rho} \psi_{, \tau} \psi_{, \rho}\right) d_{\alpha \sigma} .
$$


On setting $\psi=\log \omega$, and using the fact that $d^{\sigma \tau} d_{\alpha \sigma}=\delta^{r}{ }_{\alpha}$, there results or

$$
d^{\gamma \sigma} \omega_{, \sigma \alpha}+d^{\gamma \sigma}{ }_{, \alpha} \omega, g^{\sigma \gamma} d_{\alpha \sigma} \omega=0 \quad(\gamma \neq \alpha)
$$

$$
\left(d^{\gamma{ }^{\sigma} \omega, \sigma}\right)_{, \alpha}+g^{\sigma \gamma} d_{\alpha \sigma} \omega=0 \quad(\gamma \neq \alpha) .
$$

$A$ solution $\omega\left(u^{1}, u^{2}\right)$ of the two differential equations (25) determines the parameters $h^{\sigma}$ in (24) of a congruence whose intersector net on $S$ is parametric.

It is to be required next that the parametric intersector net be the lines of curvature net on the surface $S$. For this, it is necessary and sufficient that $g_{12}=d_{12}=0$. In this case, both of equations (25) reduce to the single equation of Laplace

$$
\left(d^{\gamma \sigma} \omega, \sigma\right)_{, \alpha}=0
$$$$
(\gamma \neq \alpha)
$$

Equation (26) may be expanded into the form

$$
d^{\gamma \sigma} \partial^{2} \omega / \partial u^{\alpha} \partial u^{\sigma}+\left(d_{, \alpha}^{\gamma \sigma}-d^{\gamma \rho} \Gamma_{\alpha \rho}^{\sigma}\right) \partial \omega / \partial u^{\sigma}=0,
$$

and this, in turn, on using

$$
d_{, \alpha}^{\gamma \sigma}=\partial d^{\gamma \sigma} / \partial u^{\alpha}+d^{\rho \sigma} \Gamma_{\rho \alpha}^{\gamma}+d^{\gamma \rho} \Gamma_{\rho \alpha}^{\sigma},
$$

takes the form

$$
d^{\gamma \sigma} \partial^{2} \omega / \partial u^{\alpha} \partial u^{\sigma}+\left(\partial d^{\gamma \sigma} / \partial u^{\alpha}+d^{\rho \sigma} \Gamma^{\gamma}{ }_{\rho \alpha}\right) \partial \omega / \partial u^{\sigma}=0 \quad(\gamma \neq \alpha) .
$$

Use of the Codazzi equations, ${ }^{4}$ together with $g_{12}=d_{12}=0$, allows equation (27) to be written out in the form

$$
2 \frac{\partial^{2} \omega}{\partial u^{1} \partial u^{2}}=\frac{d_{22}}{g_{22} d_{11}} \cdot \frac{\partial g_{11}}{\partial u^{2}} \cdot \frac{\partial \omega}{\partial u^{1}}+\frac{d_{11}}{g_{11} d_{22}} \cdot \frac{\partial g_{22}}{\partial u^{1}} \cdot \frac{\partial \omega}{\partial u^{2}} .
$$

Thus, it can be concluded that the exhibition of a congruence whose developables intersect a given surface in its lines of curvature depends upon finding a solution of equation (28). After $h^{\sigma}$ are found from equations (24), $p^{\gamma}$ and $q$ are determined by the relations $h^{\sigma} \equiv p^{\sigma} / q$ and equation (3).

To the solution $\omega\left(u^{1}, u^{2}\right) \equiv$ constant, of equation (28), there corresponds the congruence of normals to the surface $S$.

It should be oberved that if $g_{11}$ is a function of $u^{1}$ alone, or if $g_{22}$ is a function of $u^{2}$ alone, then a solution of equation (28) can be obtained by quadratures.

\section{Coincidence of the intersector net with the asymptotic net on}

\footnotetext{
${ }^{4}$ Eisenhart, loc. cit. p. 230.
} 
the surface. The intersector net may be required to coincide with a net other than the lines of curvature. For example, in case the asymptotic net on $S$ is parametric, $d_{11}=d_{22}=0$, so that if the intersector net is required to coincide with the asymptotic net, equations (25) become

$$
\left(d^{\gamma \alpha} \omega_{, \alpha}\right)_{, \alpha}+g^{\gamma \gamma} d_{\alpha \gamma} \omega=0 \quad(\gamma \neq \alpha ; \alpha, \gamma \text { not summed }) .
$$

On putting $\gamma=2, \alpha=1$, and making use of the Codazzi relation

$$
\partial d_{12} / \partial u^{1}=d_{12}\left(\Gamma_{11}^{1}-\Gamma_{12}^{2}\right),
$$

there results from equations (29)

$$
\partial^{2} \omega / \partial u^{1} \partial u^{1}+\Gamma^{1}{ }_{11} \partial \omega / \partial u^{1}-\Gamma^{2}{ }_{11} \partial \omega / \partial u^{2}+g^{22}\left(d_{12}\right)^{2} \omega=0,
$$

and similarly, with $\gamma=1, \alpha=2$,

$$
\partial^{2} \omega / \partial u^{2} \partial u^{2}-\Gamma^{1}{ }_{22} \partial \omega / \partial u^{1}+\Gamma_{22}^{2} \partial \omega / \partial u^{2}+g^{11}\left(d_{12}\right)^{2} \omega=0 .
$$

To a solution $\omega\left(u^{1}, u^{2}\right)$ of the last two equations there corresponds a congruence $\Lambda$ for which the developables intersect the surface $S$ in its asymptotic curves.

UNIVERSITY OF OKLAHOMA

\section{INTEGRAL DISTANCES}

\section{PAUL ERDÖS}

In a note under the same title (Bull. Amer. Math. Soc. vol. 51 (1945) pp. 598-600) it was shown that there does not exist in the plane an infinite set of noncollinear points with all mutual distances integral.

It is possible to give a shorter proof of the following generalization: if $A, B, C$ are three points not in line and $k=[\max (A B, B C)]$, then there are at most $4(k+1)^{2}$ points $P$ such that $P A-P B$ and $P B-P C$ are integral. For $|P A-P B|$ is at most $A B$ and therefore assumes one of the values $0,1, \cdots, k$, that is, $P$ lies on one of $k+1$ hyperbolas. Similarly $P$ lies on one of the $k+1$ hyperbolas determined by $B$ and $C$. These (distinct) hyperbolas intersect in at most $4(k+1)^{2}$ points. An analogous theorem clearly holds for higher dimensions.

UNIVERSity OF MichigAN

Received by the editors September 18, 1945. 